\title{
Prophylaxis of neutropenia with mecapegfilgrastim in patients with non-myeloid malignancies: a real-world study
}

\author{
Jun $\mathrm{Ma}^{1}$, Huiqiang Huang ${ }^{2}$, Peifen $\mathrm{Fu}^{3}$, Nong Xu${ }^{4}$, Chenyu Mao ${ }^{4}$, Gang Cheng ${ }^{5}$, Haijiao Yan $^{6}$, \\ Yongqing $\mathrm{Li}^{7}$, Yanxia $\mathrm{Shi}^{8}$, Yongsheng Wang ${ }^{9}$, Yumin Yao ${ }^{10}$, Liang Chen ${ }^{11}$, Yong Chen ${ }^{12}$, Ningling Zhang ${ }^{13}$, \\ Guifang Zhang ${ }^{14}$, Zhangxia Ren ${ }^{15}$, Zengjun Li ${ }^{16}$, Lihua Song ${ }^{17}$, Ruihua Xu ${ }^{18}$, Shukui Qin ${ }^{19}$
}

${ }^{1}$ Harbin Institute of Hematology and Oncology, Harbin, China; ${ }^{2}$ Department of Internal Medicine IV, Sun Yat-sen University Cancer Center, Guangzhou, China; ${ }^{3}$ Department of Breast Surgery, The First Affiliated Hospital of Zhejiang University School of Medicine, Hangzhou, China; ${ }^{4}$ Department of Medical Oncology, The First Affiliated Hospital of Zhejiang University School of Medicine, Hangzhou, China; ${ }^{5}$ Department of Oncology, Bozhou People's Hospital, Bozhou, China; 'Department of Oncology, The First People's Hospital of Changzhou, Changzhou, China; ${ }^{7}$ Department of Breast Surgery II, Shandong Provincial Cancer Hospital, Jinan, China; ${ }^{8}$ Department of Internal Medicine, Sun Yat-sen University Cancer Center, Guangzhou, China; ${ }^{9}$ Department of Breast Surgery I, Shandong Provincial Cancer Hospital, Jinan, China; ${ }^{10}$ Department of Breast and Thyroid, Liaocheng People's Hospital, Liaocheng, China; ${ }^{11}$ Department of gynecology I, Shandong Provincial Cancer Hospital, Jinan, China; ${ }^{12}$ Department of Radiology, The First Affiliated Hospital, Sun Yat-sen University, Guangzhou, China; ${ }^{13}$ Department of Oncology, Affiliated Hospital of North Sichuan Medical College, Nanchong, China; ${ }^{14}$ Department of Oncology, Xinxiang Central Hospital, Xinxiang, China; ${ }^{15}$ Department of Burns Plastic Surgery, Breast and Thyroid Surgery, Guang'an People's Hospital, Guang'an, China; ${ }^{16}$ Department of Lymphatic Hematology, Shandong Provincial Cancer Hospital, Jinan, China; ${ }^{17}$ Department of Breast Medicine, Shandong Provincial Cancer Hospital, Jinan, China; ${ }^{18}$ Department of Medical Oncology, Sun Yat-sen University Cancer Center, Guangzhou, China; ${ }^{19}$ Qinhuai Medical Area, Eastern Theater General Hospital of PLA China, Nanjing, China

Contributions: (I) Conception and design: J Ma, R Xu, S Qin; (II) Administrative support: J Ma, R Xu, S Qin; (III) Provision of study materials or patients: All authors; (IV) Collection and assembly of data: All authors; (V) Data analysis and interpretation: J Ma, R Xu, S Qin; (VI) Manuscript writing: All authors; (VII) Final approval of manuscript: All authors.

Correspondence to: Shukui Qin. Qinhuai Medical Area, Eastern Theater General Hospital of PLA China, Nanjing, China. Email: qinsk@csco.org.cn; Ruihua Xu. Department of Medical Oncology, Sun Yat-sen University Cancer Center, Guangzhou, China. Email: xurh@sysucc.org.cn.

Background: Chemotherapy-induced neutropenia is commonly encountered in clinical practice. The management of neutropenia has been evolving from short-acting granulocyte colony-stimulating factors (G-CSFs) to long-acting G-CSFs. However, an evaluation of the safety and effectiveness of long-acting G-CSFs in clinical practice is still lacking.

Methods: This multicenter, non-interventional study was aimed at exploring the safety and effectiveness of mecapegfilgrastim in different cancer patients in China. All patients provided written informed consent prior to the study and were treated according to routine clinical practice. Different prophylactic strategies (primary or secondary prophylaxis) were also compared.

Results: This study included 638 patients from May 2019 to November 2020. More than half of the participants were breast cancer patients. The mean age of all the patients was 56 years. White blood cell increase $(6.2 \%)$ was the most frequently reported adverse event (AE) possibly related to the study drug. No unexpected AEs were reported. Grade $\geq 3$ neutropenia in chemotherapy treatment cycle 1 was reported in $36(5.6 \%)$ patients. Incidence of grade $\geq 3$ neutropenia in cycle 1 in the primary and secondary prophylaxis subgroups were of $4.3 \%$ and $9.2 \%$, respectively. A decreasing trend of severe neutropenia incidence was observed from cycle 1 to cycle 4.

Conclusions: Mecapegfilgrastim was generally well tolerated, and no unexpected AEs were observed in this study. Primary administration of mecapegfilgrastim led to a lower incidence of neutropenia than did secondary administration. Continuous administration of mecapegfilgrastim could keep the incidence of neutropenia to a relatively low level.

Keywords: Mecapegfilgrastim; granulocyte colony-stimulating factor; neutropenia; real-world 
Submitted May 10, 2021. Accepted for publication May 21, 2021.

doi: 10.21037/atm-21-2449

View this article at: http://dx.doi.org/10.21037/atm-21-2449

\section{Introduction}

Chemotherapy-induced neutropenia, characterized as a decreased absolute neutrophil count (ANC) with or without fever, is frequently observed in non-myeloid malignancies (1). Neutropenia is one of serious chemotherapy-related hematological adverse events (AEs) that could lead to dose reductions/delays and may compromise treatment outcomes (2-4). Chemotherapy-induced neutropenia also incurs a great economic burden on cancer patients via hospitalization and follow-up care, and may even by lifethreatening $(5,6)$.

Granulocyte colony-stimulating factor (G-CSF) is one of the hematopoietic growth factors that facilitates the differentiation of committed granulocyte progenitors into mature granulocytes (e.g., neutrophils) (7). It has been widely used to prevent neutropenic complications of myelosuppressive chemotherapy. G-CSF was first developed as a short-acting formula. As shorting-acting G-CSF requires daily administration during chemotherapy, the long-acting formula, a pegylated G-CSF, was developed to overcome this inconvenience. Mecapegfilgrastim (HHPG$19 \mathrm{~K}$ ) is a novel long-acting G-CSF which was approved by the Chinese National Medical Products Administration (NMPA) in 2018. With the nature of a pegylated G-CSF, the half-life was greatly prolonged comparing to shortacting G-CSF. The administration of mecapegfilgrastim was only required once in each chemotherapy cycle, as a consequence, the compliance of patients was better than patients using short-acting G-CSF which required to be administrated each day during chemotherapy. The efficacy and safety profiles of mecapegfilgrastim has been investigated in 2 pivotal phase 3 randomized clinical trials $(8,9)$. The results showed that mecapegfilgrastim was non-inferior and even superior to short-acting G-CSF in reducing the incidence/duration of severe neutropenia in breast cancer and non-small cell lung cancer patients. Furthermore, the safety profiles of mecapegfilgrastim and filgrastim are similar $(8,9)$.

Despite the efficacy and safety of mecapegfilgrastim in prophylaxis of neutropenia being studied in several trials (8-10), the effectiveness and safety have not been well studied under a real-world setting in a large group of patients. Therefore, we initiated this real-world study to explore the effectiveness and safety profile of mecapegfilgrastim in patients with different types of cancer.

We present the following article in accordance with the STROBE reporting checklist (available at http://dx.doi. org/10.21037/atm-21-2449).

\section{Methods}

\section{Study design}

This was a prospective, multicenter, non-interventional, real-world study. The enrollment of patients began from May 2019. All patients provided written informed consent prior to the study and were treated in accordance with the Declaration of Helsinki (as revised in 2013) and routine clinical practice. This study was approved by the ethics committee of all study centers (No. 2019-006). The commercial electronic data capture system was applied to collect data. Source document verification was also conducted.

\section{Eligible criteria}

Patients were included if they were at least 18 years old, signed the informed consent forms, were pathologically or cytohistologically confirmed to have non-myeloid malignancy (including solid tumor and hematological tumor), and were considered able to tolerate mecapegfilgrastim by investigators. Patients were excluded if they were females who were pregnant or breastfeeding, hypersensitive to mecapegfilgrastim, recombinant human granulocyte colony-stimulating factor (rhG-CSF), pegylated rhG-CSF (PEG-rhG-CSF) or other similar biological agents; or were otherwise deemed by investigators to be not eligible for the study.

\section{Outcomes}

The primary outcome was incidence of any AEs, including changes in clinical laboratory values, vital signs, and physical examinations. The grading of the AEs was done according to National Cancer Institute - Common Terminology 
Criteria for Adverse Events (version 5.0). The association between AEs and the investigated drug was evaluated by investigators.

The secondary outcomes were the incidence of grade $\geq 3$ and grade 4 neutropenia (defined as ANC less than $1.0 \times 10^{9} / \mathrm{L}$ and less than $0.5 \times 10^{9} / \mathrm{L}$, respectively) in cycle 1 and the incidence of grade $\geq 3$ and grade 4 neutropenia from cycle 1 to cycle 4 .

\section{Study drug administration}

The administration of study drug followed the routine clinical practice. In general, patients received mecapegfilgrastim subcutaneously by fixed dose $(6 \mathrm{mg})$ or by weight $(100 \mu \mathrm{g} / \mathrm{kg})$ up to $1-3$ days after each chemotherapy cycle. The National Comprehensive Cancer Network (NCCN) guideline for the management of neutropenia (version 1.2019) was followed to guide the investigators' practice.

\section{Statistical analysis}

This study included patients who completed the study from May 2019 to November 2020.

The full analysis set (FAS) was defined as enrolled patients who received mecapegfilgrastim at least once. The modified full analysis set (mFAS) was defined as the patients in FAS who had completed at least 4 treatment cycles and documented the ANC. The safety set (SS) was defined as the patients received mecapegfilgrastim at least once and had safety records.

The baseline characteristics of patients were presented with descriptive statistics in FAS. The primary outcome was evaluated in SS. The secondary outcomes were evaluated in FAS and mFAS.

Subgroup analysis (stratification factors: prophylaxis, primary or secondary; 1-, 2- , 3-, or 4-week chemotherapy) was conducted for the incidence of neutropenia in cycle 1 .

All statistical analyses were performed using SAS version 9.4 (SAS Institute, Cary, NC, USA).

\section{Results}

\section{Patients}

From May 2019 to November 2020, 638 patients received the study drug at least once, and those that completed the study were included in the FAS. A further 613 patients from the FAS who had safety records were included in the SS, while 175 patients from the FAS who had an ANC documentation of 4 cycles were considered for the mFAS and used to explore the effectiveness trend from chemotherapy cycle 1 to cycle 4 .

Most of the patients were female (68.5\%), and about half of the participants were breast cancer patients. The mean age was 55.7 years, and $26.6 \%$ were 65 years or older. Patients with an Eastern Cooperative Oncology Group (ECOG) performance status of $0-1$ constituted $92.3 \%$ of the population, and $77.3 \%$ of patients received mecapegfilgrastim as primary prophylaxis. The detailed baseline characteristics are presented in Table 1 .

\section{Safety}

All patients in the SS $(n=613)$ experienced an AE at least once. Adverse drug reactions (ADRs), as identified by investigators, were reported in 133 patients $(21.7 \%)$. The most frequently reported ADR was white blood cell count increase $(6.2 \%)$. White blood cell count decrease, nausea, and vomiting were reported as ADRs in about $3 \%$ patients. Neutrophil count increase and anemia were reported as ADRs in about $2 \%$ patients (Table 2). No grade 4 or higher ADRs were reported. In the SS, 16 (2.6\%) patients reported serious AEs, with 1 being considered possibly related to the study drug.

\section{Effectiveness}

\section{Incidence of neutropenia}

During the first chemotherapy cycle, 36 (5.6\%) patients experienced grade $\geq 3$ neutropenia with 10 (1.6\%) being grade 4 neutropenia. From cycle 1 to cycle 4 , the incidence of grade 3 neutropenia had an overall decreasing trend (5.7\%, 2.9\%, 2.9\% and 1.7\%, respectively). A trend toward a lower incidence of grade 4 neutropenia was also observed with the continuous administration of mecapegfilgrastim (from cycle 1 to cycle $4,4 \%, 0.6 \%, 1.7 \%$ and $1.7 \%$, respectively) (Figure 1).

\section{Subgroup analysis of effectiveness}

In the subgroup analysis, $21(4.3 \%)$ patients in the primary prophylaxis group and $13(9.2 \%)$ patients in secondary prophylaxis group experienced grade $\geq 3$ neutropenia in cycle 1 .

Meanwhile, $6(1.2 \%)$ patients in the primary prophylaxis group and $4(2.8 \%)$ patients in secondary prophylaxis group 
Table 1 Characteristics of the participants

\begin{tabular}{|c|c|}
\hline Characteristic & FAS $(n=638)$ \\
\hline \multicolumn{2}{|l|}{ Sex, n (\%) } \\
\hline Male & $200(31.3)$ \\
\hline Female & 437 (68.5) \\
\hline Missing & $1(0.2)$ \\
\hline Age, mean \pm SD & $55.7(12.23)$ \\
\hline \multicolumn{2}{|l|}{ Age group, n (\%) } \\
\hline$<65$ & $466(73.0)$ \\
\hline$\geq 65$ & $170(26.6)$ \\
\hline Missing & $2(0.4)$ \\
\hline \multicolumn{2}{|l|}{ ECOG PS, n (\%) } \\
\hline $0-1$ & 589 (92.3) \\
\hline$\geq 2$ & $4(0.6)$ \\
\hline Missing & $45(7.1)$ \\
\hline \multicolumn{2}{|l|}{ Prior G-CFS, n (\%) } \\
\hline Yes & $142(22.3)$ \\
\hline No & $493(77.3)$ \\
\hline Missing & $3(0.4)$ \\
\hline \multicolumn{2}{|l|}{ Prophylaxis strategy, n (\%) } \\
\hline Primary & $493(77.3)$ \\
\hline Secondary & $142(22.3)$ \\
\hline Missing & $3(0.4)$ \\
\hline \multicolumn{2}{|l|}{ Radiotherapy history, n (\%) } \\
\hline Yes & 66 (10.3) \\
\hline No & $566(88.7)$ \\
\hline Missing & $6(1.0)$ \\
\hline \multicolumn{2}{|l|}{ Cycle length, n (\%) } \\
\hline 1 week & $20(3.1)$ \\
\hline 2 weeks & $41(6.4)$ \\
\hline 3 weeks & $407(63.8)$ \\
\hline 4 weeks & $14(2.3)$ \\
\hline Missing & $156(24.4)$ \\
\hline \multicolumn{2}{|l|}{ Study drug dosage, n (\%) } \\
\hline Fixed dosage (6 mg) & $626(98.1)$ \\
\hline Weight-adjusted dosage $(100$ g/kg) & $12(1.9)$ \\
\hline
\end{tabular}

Table 1 (continued)
Table 1 (continued)

\begin{tabular}{lc}
\hline Characteristic & FAS $(\mathrm{n}=638)$ \\
\hline Cancer type, $\mathrm{n}(\%)$ & \\
Solid tumor: & \\
Breast cancer & $323(50.6)$ \\
Colorectal cancer & $51(8.0)$ \\
Non-small cell lung cancer & $43(6.7)$ \\
Gastric cancer & $35(5.5)$ \\
Small cell lung cancer & $33(5.2)$ \\
Esophageal cancer & $22(3.4)$ \\
Ovarian cancer & $16(2.5)$ \\
Pancreatic cancer & $13(2.0)$ \\
Nasopharyngeal cancer & $9(1.4)$ \\
Cervical cancer & $7(1.1)$ \\
Head and neck cancer & $4(0.6)$ \\
Hematological tumor: & $137(21.5)$ \\
DLBCL & $209(32.8)$ \\
Other B-cell lymphoma & $133(20.8)$ \\
T-cell lymphoma & $5(20.8)$ \\
Others & $5(0.8)$ \\
Cancer stage & \\
I & \\
\hline III & \\
\hline
\end{tabular}

ECOG PS, Eastern Cooperative Oncology Group performance status; FAS, full analysis set; G-CFS, granulocyte colonystimulating factor; DLBCL, diffused large-B cell lymphoma.

Table 2 The most frequently reported adverse events possibly related to study drug

\begin{tabular}{lc}
\hline Adverse events & SS (n=613) \\
\hline White blood cell count increase, $\mathrm{n}(\%)$ & $38(6.2)$ \\
White blood cell count decrease, $\mathrm{n}(\%)$ & $19(3.1)$ \\
Nausea, $\mathrm{n}(\%)$ & $20(3.3)$ \\
Vomiting, $\mathrm{n}(\%)$ & $19(3.1)$ \\
Anemia, $\mathrm{n}(\%)$ & $13(2.1)$ \\
Neutrophil count increase, $\mathrm{n}(\%)$ & $14(2.3)$ \\
\hline
\end{tabular}

SS, safety set. 


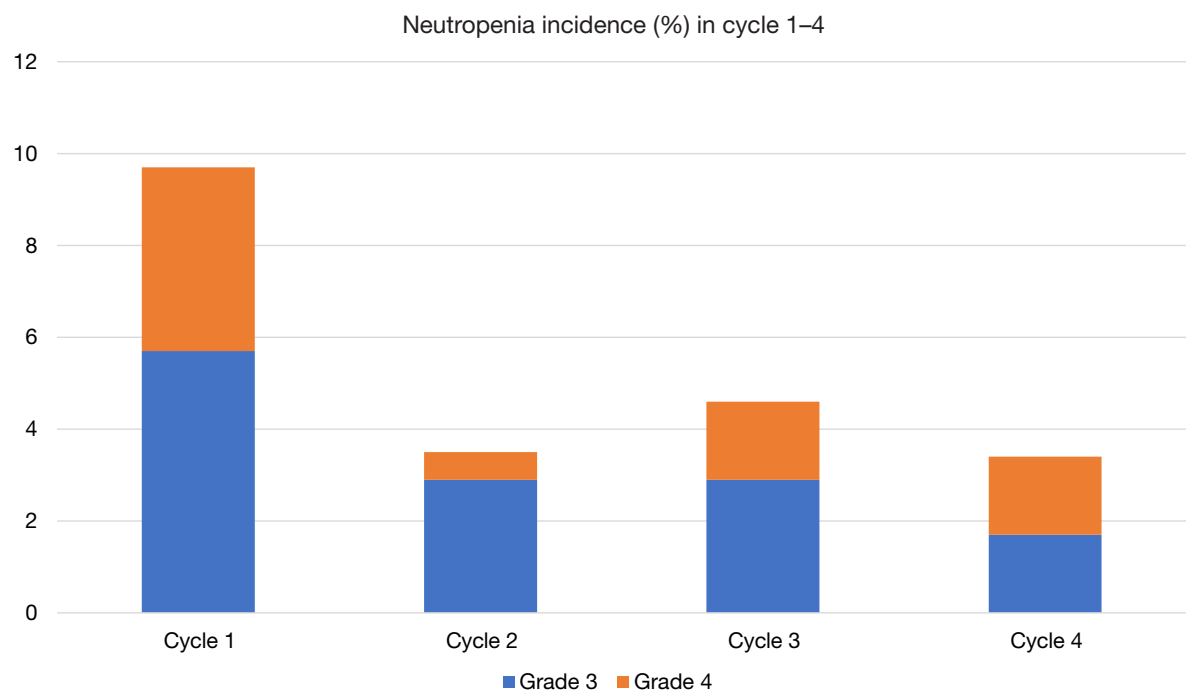

Figure 1 Incidence of neutropenia from cycle 1 to cycle 4 in participants with absolute neutrophil count records in all 4 cycles (mFAS, n=175).

Table 3 Effectiveness by different subgroups in chemotherapy cycle 1

\begin{tabular}{lcc}
\hline \multirow{2}{*}{ Treatment } & \multicolumn{2}{c}{ Endpoints } \\
\cline { 2 - 3 } Prophylaxis strategy & Grade $\geq 3$ neutropenia, $n(\%)$ & Grade 4 neutropenia, $n(\%)$ \\
Primary $(n=493)$ & $21(4.3)$ & $4(1.2)$ \\
Secondary $(n=142)$ & $13(9.2)$ & $3(15)$ \\
Chemotherapy & & 0 \\
1 week $(n=20)$ & $3(15)$ & $6(1.5)$ \\
2 weeks $(n=41)$ & $1(2.4)$ & 0 \\
3 weeks $(n=407)$ & $28(6.9)$ & $1(7.1)$ \\
4 weeks $(n=14)$ & & \\
\hline
\end{tabular}

experienced grade 4 neutropenia in cycle 1 . Patients with a 1-week chemotherapy plan had the highest incidence of neutropenia (Table 3). Approximately $7 \%$ of patients with non-small cell lung cancer had grade $\geq 3$ neutropenia in cycle 1 , which was the highest proportion according to cancer type and was followed by breast cancer (6.2\%), small cell lung cancer (6.1\%), hematological cancer (4.8\%), colorectal cancer $(3.9 \%)$, and gastric cancer $(2.9 \%)$.

\section{Discussion}

The efficacy and safety of mecapegfilgrastim had been explored in non-small cell lung cancer patients and breast cancer patients previously. In this study, we investigated the tolerability and effectiveness of mecapegfilgrastim in various cancer patients in routine clinical practice. Administration of mecapegfilgrastim in real-world setting could reduce the incidence of grade $\geq 3$ neutropenia and there were no unexpected AEs were reported.

In a phase 2 study of breast cancer patients treated with $100 \mu \mathrm{g} / \mathrm{kg}$ of mecapegfilgrastim, it was reported that leukocytopenia occurred in $10 \%$ patients, while neutrophilia, leukocytosis, and thrombocytosis occurred in $6.7 \%$ patients; the non-hematologic AEs with an incidence of greater than 5\% were nausea, vomiting, anorexia and myalgia (10). A pivotal phase 3 study of mecapegfilgrastim in breast cancer patients found that the most frequently reported treatment-related AEs were hemoglobin decline (12.6\% and 13.6\%) and fatigue 
$(15.3 \%$ and $13.6 \%)$ in a $100 \mu \mathrm{g} / \mathrm{kg}$ group and a fixed dose $6 \mathrm{mg}$ group respectively (9). Another pivotal phase 3 study of mecapegfilgrastim in non-small cell lung cancer patients found leukocytosis $(8.5 \%$ and $2.1 \%)$ and fatigue $(4.3 \%$ and $6.3 \%$ ) in a 100 the $\mu \mathrm{g} / \mathrm{kg}$ group and fixed dose $6 \mathrm{mg}$ group, respectively (8). In the previous key trials of breast cancer patients, the most frequently reported $\mathrm{AE}$ possibly related to pegfilgrastim was skeletal/bone pain (more than $25 \%$ ) $(11,12$ ). Our real-world study found that the most frequently reported AE possibly related to the study drug was white blood cell count increase. Only $1(0.2 \%)$ patient reported back pain, representing a lower incidence of back pain (less than $0.9 \%$ ) than that reported in the previous phase 3 breast cancer trial. There were 137 (22.3\%) patients who received an analgesic drug combination, which could have been the cause of the relatively low incidence of bone pain.

In a phase 3 study of mecapegfilgrastim. about $50 \%$ and $30 \%$ breast cancer patients experienced grade $\geq 3$ and grade 4 neutropenia, respectively, after administration of mecapegfilgrastim in cycle 1 . This was probably related to the highly toxic chemotherapy (Anthracyclines and Taxane, AT or Adriamycin and Cyclophosphamide, AC) used for these patients (9). There was also decreasing trend of incidence of neutropenia from cycle 1 to cycle 4 reported in breast cancer patients after administration of mecapegfilgrastim (9). This trend was also reported in the 2 phase 3 trials in breast cancer patients given pegfilgrastim $(11,12)$. In our study, we did not find a high incidence of neutropenia in cycle 1 as compared to the previous phase 3 breast cancer trial (9). The chemotherapy used for breast cancer patients in this study was more diverse and of lower toxicity compared with the chemotherapy (AC or AT) used several years ago. The incidence of grade $\geq 3$ neutropenia in cycle 1 of non-small cell lung cancer patients was similar to that of a pivotal phase 3 trial (8), while a similar decreasing trend of neutropenia incidence rate from cycle 1 to cycle 4 was identified in these key trials and in this real-world study.

In terms of the prophylactic strategy of G-CSF, primary or secondary prophylaxis has been explored in several studies. A real-world study in Belgium and Luxembourg found that patients receiving lipegfilgrastim had a lower incidence of grade 3 and grade 4 neutropenia than did those receiving it secondarily (13). Another prospective, non-interventional, multicenter study conducted in Germany also reported that primarily administration of lipegfilgrastim demonstrated a lower incidence of severe neutropenia compared with secondary administration (14). These findings are in line with our results.
Pegfilgrastim has not been recommended for weekly administration in patients treated with cytotoxic chemotherapy, as there is insufficient supporting data (15). The high incidence of neutropenia in patients of 1-week chemotherapy was observed in this real-world study and is likely linked with the mechanism of long-acting G-CSF.

There were several limitations to our research that should be noted. First, the number of patients included for analysis was still relatively small. Second, all analyses were descriptive, and no formal statistical assumptions were applied. Nonetheless, this study provides support for continuing this line of research and insights into clinical practice.

\section{Conclusions}

Mecapegfilgrastim was well tolerated in different cancer patients, and no unexpected AEs were observed in this real-world setting. Primary prophylactic administration of mecapegfilgrastim could lower the incidence of neutropenia as compared to secondary usage. Administration of mecapegfilgrastim continuously could keep the incidence of neutropenia at a relatively low level.

\section{Acknowledgments}

Part of this work had been accepted by ASCO 2021 annual meeting as an abstract (publish only).

Funding: Jiangsu Hengrui Pharmaceuticals Co., Ltd., Shanghai, China.

\section{Footnote}

Reporting Checklist: The authors have completed the STROBE reporting checklist. Available at http://dx.doi. org/10.21037/atm-21-2449

Data Sharing Statement: Available at http://dx.doi. org/10.21037/atm-21-2449

Conflicts of Interest: All authors have completed the ICMJE uniform disclosure form (available at http://dx.doi. org/10.21037/atm-21-2449). The authors have no conflicts of interest to declare.

Ethical Statement: The authors are accountable for all aspects of the work in ensuring that questions related to the accuracy or integrity of any part of the work are 
appropriately investigated and resolved. This study was approved by the ethics committee of all study centers (No. 2019-006). The commercial electronic data capture system was applied to collect data. All patients provided written informed consent prior to the study and were treated in accordance with the Declaration of Helsinki (as revised in 2013) and routine clinical practice.

Open Access Statement: This is an Open Access article distributed in accordance with the Creative Commons Attribution-NonCommercial-NoDerivs 4.0 International License (CC BY-NC-ND 4.0), which permits the noncommercial replication and distribution of the article with the strict proviso that no changes or edits are made and the original work is properly cited (including links to both the formal publication through the relevant DOI and the license). See: https://creativecommons.org/licenses/by-nc-nd/4.0/.

\section{References}

1. Crawford J, Dale DC, Kuderer NM, et al. Risk and timing of neutropenic events in adult cancer patients receiving chemotherapy: the results of a prospective nationwide study of oncology practice. J Natl Compr Canc Netw 2008;6:109-18.

2. Lyman GH. Impact of chemotherapy dose intensity on cancer patient outcomes. J Natl Compr Canc Netw 2009;7:99-108.

3. Bonadonna G, Valagussa P, Moliterni A, et al. Adjuvant cyclophosphamide, methotrexate, and fluorouracil in nodepositive breast cancer: the results of 20 years of follow-up. N Engl J Med 1995;332:901-6.

4. Lyman GH, Dale DC, Friedberg J, et al. Incidence and predictors of low chemotherapy dose-intensity in aggressive non-Hodgkin's lymphoma: a nationwide study. J Clin Oncol 2004;22:4302-11.

5. Weycker D, Malin J, Edelsberg J, et al. Cost of neutropenic complications of chemotherapy. Ann Oncol 2008;19:454-60.

6. Pizzo PA. Management of fever in patients with cancer and treatment-induced neutropenia. N Engl J Med 1993;328:1323-32.

7. Bennett CL, Djulbegovic B, Norris LB, et al. Colonystimulating factors for febrile neutropenia during cancer therapy. N Engl J Med 2013;368:1131-9. Erratum in: N Engl J Med 2013;369:293.

8. Zhou C, Huang Y, Wang D, et al. A Randomized Multicenter Phase III Study of Single Administration of
Mecapegfilgrastim (HHPG-19K), a Pegfilgrastim Biosimilar, for Prophylaxis of Chemotherapy-Induced Neutropenia in Patients With Advanced Non-Small-Cell Lung Cancer (NSCLC). Clin Lung Cancer 2016;17:119-27.

9. Xu F, Zhang Y, Miao Z, et al. Efficacy and safety of mecapegfilgrastim for prophylaxis of chemotherapy-induced neutropenia in patients with breast cancer: a randomized, multicenter, active-controlled phase III trial. Ann Transl Med 2019;7:482. Erratum in: Ann Transl Med 2020;8:660.

10. Wang T, Wu B, Hu X, et al. A randomized multicenter phase II trial of mecapegfilgrastim single administration versus granulocyte colony-stimulating growth factor on treating chemotherapy-induced neutropenia in breast cancer patients. Ann Transl Med 2019;7:196.

11. Green MD, Koelbl H, Baselga J, et al. A randomized double-blind multicenter phase III study of fixed-dose single-administration pegfilgrastim versus daily filgrastim in patients receiving myelosuppressive chemotherapy. Ann Oncol 2003;14:29-35.

12. Holmes FA, O'Shaughnessy JA, Vukelja S, et al. Blinded, randomized, multicenter study to evaluate single administration pegfilgrastim once per cycle versus daily filgrastim as an adjunct to chemotherapy in patients with high-risk stage II or stage III/IV breast cancer. J Clin Oncol 2002;20:727-31.

13. Fontaine C, Claes N, Graas MP, et al. Effect of lipegfilgrastim administration as prophylaxis of chemotherapy-induced neutropenia on dose modification and incidence of neutropenic events: real-world evidence from a non-interventional study in Belgium and Luxembourg. Acta Clin Belg 2021;76:10-5.

14. Kurbacher CM, Fietz T, Diel IJ, et al. NADIR: A NonInterventional Study on the Prophylaxis of ChemotherapyInduced Neutropenia Using Lipegfilgrastim - First Interim Analysis. Oncol Res Treat 2015;38:221-9.

15. Becker PS, Griffiths EA, Alwan LM, et al. NCCN Guidelines Insights: Hematopoietic Growth Factors, Version 1.2020. J Natl Compr Canc Netw 2020;18:12-22.

(English Language Editor: J. Gray)

Cite this article as: $\mathrm{Ma} \mathrm{J}$, Huang $\mathrm{H}, \mathrm{Fu} \mathrm{P}, \mathrm{Xu} \mathrm{N}$, Mao C, Cheng G, Yan H, Li Y, Shi Y, Wang Y, Yao Y, Chen L, Chen Y, Zhang N, Zhang G, Ren Z, Li Z, Song L, Xu R, Qin S. Prophylaxis of neutropenia with mecapegfilgrastim in patients with non-myeloid malignancies: a real-world study. Ann Transl Med 2021;9(10):893. doi: 10.21037/atm-21-2449 